\title{
Transient simulation of very large scale models using Krylov subspace-based model order reduction
}

\author{
O. Macchion ${ }^{1}$, A. Suleng ${ }^{1}$, M. Mork ${ }^{1} \&$ J. Sindler ${ }^{2}$ \\ ${ }^{I}$ Flow Assurance Department, Production Performance Services, \\ FMC Kongsberg Subsea AS, Norway \\ ${ }^{2}$ Czech Technical University, Czech Republic
}

\begin{abstract}
The scale of large finite element (FE) models may nowadays easily exceed $10^{7}$ or even $10^{8}$ degrees of freedom (DOF), leading to excessive calculation times when performing transient simulations. Such long simulation times hinder effective structural or thermal design and optimization and make any engineering insight into a problem difficult. The Krylov subspace-based model order reduction (MOR) is a reduction method based on projection of a discretized model onto a lower dimension subspace. The paper presents a methodology based on this method in the context of thermal transient simulation of a large scale subsea equipment FE model. The finite element model mesh size exceeds $30 \times 10^{6} \mathrm{DOFs}$. The problem has nonzero initial conditions (ICs) and has to be transformed into a problem with zero ICs in order to apply the Krylov based MOR. Coupling the Krylov based MOR models employs a novel technique involving coupling through their surface interfaces. The approach is compared with the solution obtained using a full FE simulation which takes about 7 days to solve with a fine time step. The results are compared using an error norm which computes maximum absolute difference of temperature fields over time taking the full FE simulation with the fine time step as a reference. The study shows that applying the proposed method using Krylov MOR for performing thermal transient simulations is valid and leads to substantial reduction of the computational time.

Keywords: model order reduction, substructuring, heat transfer.
\end{abstract}




\section{Introduction}

Oil discoveries are becoming more marginal and more challenging to develop due to e.g. deep water and challenging environments. Consequently, subsea tieback to a central processing facility is becoming increasingly attractive. Currently, subsea developments are the preferred option for many fields and often the only economically viable option. A typical subsea field includes subsea wells and a subsea production system featuring well control and commingling of flow together with flow lines and risers.

For successful operation of subsea oil and gas fields, good control of the thermal behaviour of the subsea production system, flow lines and risers is essential for stable operation and high uptime of the whole field. Therefore, the whole subsea system is designed to prevent heat loss both during normal operation and in a shutdown situation. Most of the technologies for temperature control include thermal insulation. For the subsea production system in specific, with Xmas Trees for well control, manifolds and connections, thermal insulation is the main solution.

For a typical deep-water oil field, the subsea production system must be kept warm during normal production, but also in case of a shutdown. When the well fluid is cooled at shutdown, there is a risk of forming gas hydrates, unless preventive actions are taken upfront. Gas hydrates only exist at low temperatures (typically seawater temperatures) and at high pressure, and can block the whole subsea production system if not avoided. The thermal insulation is required to allow the operator of the field to take the required preventive actions before the whole field is cooled down. If no time is available, the consequence may be months of lost production due to hydrate plugs in the system. Therefore, a certain cool down time of the production fluid, typically six to thirteen hours, is required to slow down the cooling of the production fluid in case of an unplanned shutdown. For each field, this cool down time requirement is investigated and verified through thermal analyses, usually performed using FEA or CFD, in the field's design phase.

In the subsea production system there are many items, such as valves, sensors, and connections that cannot be fully covered with insulation to be able to function properly. Hence, a typical manifold may consist of well-insulated areas and areas without insulation that are draining heat out of the system. The total thermal performance is highly dominated by these uninsulated cold spots. To obtain an insulation design of the subsea production system that fulfils the field's cool down requirement, extensive thermal $3 \mathrm{D}$ modelling is required, typically of both small sensors and large manifolds. The phenomena to investigate include conduction and convection, especially natural convection, which are computationally intensive for such large models and long cool down times.

The simulations are based on numerical models discretizing the equations of mass, momentum, and enthalpy, on the 3D model generated by the designer of the system. The requirements in terms of grid resolution and time stepping needed to achieve good enough accuracy of the results lead to large models, in the range of 10 to 100 million degrees of freedom, and excessive calculation times. Most 
calculations performed within the framework of an Engineering, Procurement, and Construction (EPC) project are limited to the verification of the proposed insulation design. This goes against the demands of the industry, which today requires equipment with broadened operational envelopes to meet the increasing difficulty in extracting the oil left in the North Sea, and the deepwater fields off the coasts of Brazil and Africa.

In this context substructuring [1], and model order reduction, [2-4], appear as a means of decreasing drastically preprocessing and calculation times, and open the way to a more robust design by allowing investigation of the sensitivity of the design to input parameters. A detailed overview of available model order reduction methods can be found in [2], while recent developments of model reduction by projection on a Krylov subspace can be found in [5, 6], and [8]. One of the most important directions in the development of Krylov-based reductions is parametric model order reduction (PMOR). PMOR allows preservation of parameters which the system depends on (see [7] and [11]). Another important avenue of research pioneered in [9] and further developed in [10], is the extension of Krylov-based MOR to nonlinear systems and convective flows.

The overall aim of the present document is to establish a methodology integrating the use of substructuring and model order reduction in the simulation of the thermal cool down of a subsea manifold (see Figure 1).

This paper is organized as follows; first, the technical problem is presented in section 2. The solution methodology to be evaluated is outlined in section 3 . The results are presented in section 4, and a discussion of these is led in section 5 . Section 6 summarizes and concludes the work presented in the paper.

\section{Problem statement}

The heat transfer between the production fluid, metal bodies, and insulation layers in subsea equipment is modelled by the transient heat transfer equation,

$$
T_{t}+\frac{k}{\rho C_{p}} T_{i, i}=0,
$$

with $k, \rho, C_{p}$ being thermal conductivity in $\mathrm{Wm}^{-1} \mathrm{~K}^{-1}$, density in $\mathrm{kg} \mathrm{m}^{-3}$, and thermal capacity of the materials in $\mathrm{J} \cdot \mathrm{kg}^{-1} \cdot \mathrm{K}^{-1} \cdot T_{t}$ denotes the derivative of the temperature according to time, while $T_{i, i}$ denotes the Laplacian of the temperature field.

The convective fluid flow of the production fluid imprisoned within the piping system is modelled with a thermal conductivity high enough to account for the global effects of convection on the temperature distribution in the fluid. Obviously, no local effects or natural convection boundary layers can be modelled in this way. We choose to ignore this and will consider the application of model order reduction to convective flows in later studies.

The discretization in space of the manifold of interest (see Figure 1), is not the object of this paper. Thus Figure 2 shows an example of the type of mesh generated for this type of calculation, and is taken as the starting point of the methodology presented in section 3. In addition, the results presented in section 4 are compared to reference results obtained by solving the full problem, whose mesh is shown in Figure 2, in physical space and time. 


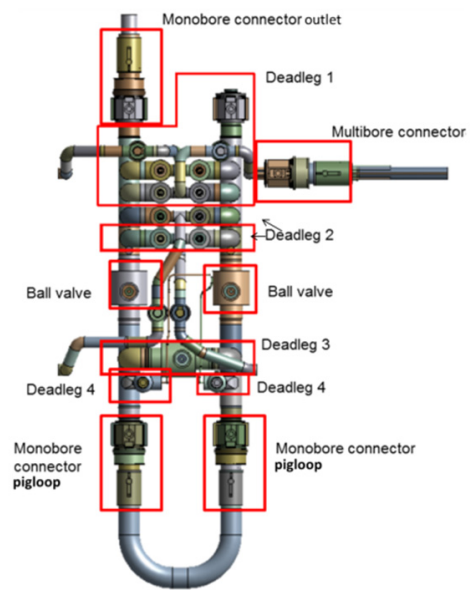

Figure 1: Manifold and its substructures.

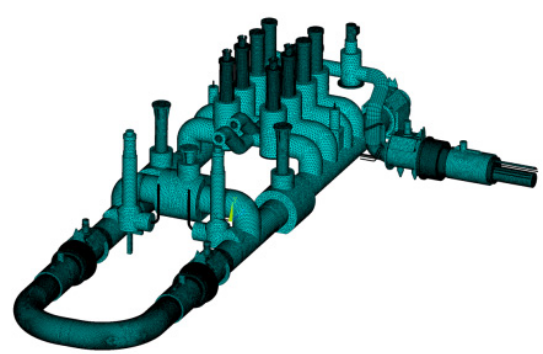

Figure 2: Manifold mesh.

Steady state heat transfer within the manifold implies a constant flow of production fluid, at a fixed temperature, in the piping system. Surfaces in contact with sea water were assigned a constant temperature of $4^{\circ} \mathrm{C}$.

Transient heat transfer within the manifold implies production shutdown, with the far boundaries modelled as adiabatic boundary conditions. This assumes that cool down is uniform on both sides of the boundary.

\section{Solution methodology}

This section presents the two main mathematical techniques that are used in this study, namely model order reduction of linear dynamical systems (see section 3.1), and coupling of substructured and reduced models of large finite element models (see section 3.2).

We consider linear time invariant (LTI) systems with zero initial conditions of the form

$$
\boldsymbol{C} \dot{\boldsymbol{T}}+\boldsymbol{K} \boldsymbol{T}=q u,
$$

where $T(t) \in \mathbb{R}^{N \times 1}$ and $u(t) \in \mathbb{R}$ denote the states and input of the system, respectively. The dynamics of the system are described by $\boldsymbol{C}, \boldsymbol{K} \in \mathbb{R}^{N \times N}$ and $\boldsymbol{q} \in$ $\mathbb{R}^{N \times 1}$. In the context of heat transfer within a subsea production system component, $\boldsymbol{C}$ is the matrix of heat capacity at the discretization nodes and $\boldsymbol{K}$ is the matrix of heat conductance between internal nodes. It is assumed that $\boldsymbol{C}$ is non-singular; $\operatorname{det}(\boldsymbol{C}) \neq 0$, which is usually the case for a mesh with non-negative volumes.

The goal of model order reduction is to approximate the large-scale system (2), e.g. with $N=10^{5} \ldots 10^{8}$, by one of much smaller dimension $n \ll N$. In projection-based model reduction, this is carried out by appropriate projection 
matrices $\boldsymbol{V}, \boldsymbol{W} \in \mathbb{R}^{N \times n}$, leading to a reduced system with state-space representation

$$
\boldsymbol{C}_{r} \dot{\boldsymbol{\tau}}+\boldsymbol{K}_{r} \boldsymbol{\tau}=\boldsymbol{q}_{r} u,
$$

where $\boldsymbol{C}_{\boldsymbol{r}}=\boldsymbol{W}^{\boldsymbol{T}} \boldsymbol{C} \boldsymbol{V}, \boldsymbol{K}_{\boldsymbol{r}}=\boldsymbol{W}^{\boldsymbol{T}} \boldsymbol{K} \boldsymbol{V}$ and $\boldsymbol{q}_{\boldsymbol{r}}=\boldsymbol{W}^{\boldsymbol{T}} \boldsymbol{q}$. The reduced system (3) is called a Petrov-Galerkin projection of (2) [2]. The generalized coordinates $\tau$ are defined as

$$
T=V \tau+\varepsilon,
$$

where $\varepsilon \in \mathbb{R}^{N \times 1}$ is the matrix of error.

\subsection{Model order reduction}

Model order reduction (MOR) aims at the approximation of large-scale dynamical systems by another model of reduced order. For the reduction of LTI systems different approaches have been shown to be well suited, such as Truncated Balanced Realization (TBR) [2] or Krylov subspace methods [3, 4].

In this study the projection matrix $\boldsymbol{V}$ (or $\boldsymbol{W}$ ) is chosen to span certain rational Krylov subspaces.

A Krylov subspace is generally defined as

$$
K^{k}(M, N)=\operatorname{span}\left\{\boldsymbol{N}, \boldsymbol{M N}, \ldots, \boldsymbol{M}^{k-1} \boldsymbol{N}\right\} .
$$

In the particular case of system (2), and after selecting a complex valued expansion point $s_{i} \in \mathbb{C}$ and a desired multiplicity $m_{i} \in \mathbb{N}^{+}$the matrices $\mathrm{N}$ and $\mathrm{M}$ read

$$
\boldsymbol{N}=\left(\boldsymbol{K}+s_{i} \boldsymbol{C}\right)^{-1} \boldsymbol{Q}, \quad \boldsymbol{M}=\left(\boldsymbol{K}+s_{i} \boldsymbol{C}\right)^{-1} \boldsymbol{C},
$$

Then the Krylov subspace reads

$$
K_{s_{i}}^{m_{i}}(\boldsymbol{K}, \boldsymbol{C})=K^{m_{i}}\left(\left(\boldsymbol{K}+s_{i} \boldsymbol{C}\right)^{-1} \boldsymbol{Q},\left(\boldsymbol{K}+s_{i} \boldsymbol{C}\right)^{-1} \boldsymbol{C}\right) .
$$

If the projection matrix $\boldsymbol{V}$ then is computed to span the union of certain Krylov subspaces,

$$
\operatorname{span}(\boldsymbol{V}) \supseteq K_{s_{i}}^{m_{i}}, \quad i=1, \ldots, r
$$

the reduced model (3) matches $m_{i}$ moments of the transfer function of (2) around the respective expansion points $s_{i}$, if $s_{i}$ is neither an eigenvalue of (2) nor an eigenvalue of (3) [2-4]. The $m_{i}$ moments are defined as the coefficients of the Taylor series expansion of the transfer function $H(s)=\left(s_{i} C+K\right) Q$ around $s_{i}$.

The projection matrix $\boldsymbol{V}$ is obtained by Arnoldi iteration, which means that $\boldsymbol{W}=\boldsymbol{V}$ due to the block Arnoldi algorithm being one sided. The first step in this process is to choose the first Arnoldi vector $v_{1}=N /\|N\|_{2}$. Then, the subsequent steps consist in a multiplication, for $j=1,2, \ldots, n$, of the current Arnoldi vector $v_{j}$ by $\boldsymbol{M}$, followed by orthonormalization of the product $M v_{j}$ against all previous Arnoldi vectors. This procedure is summarized in Algorithm 1, where the vectors $\mathrm{w}_{\mathrm{j}}$ are the intermediate un-normalized vectors. 
Algorithm 1 Arnoldi iteration (classical Gram-Schmidt variant)

$$
\begin{aligned}
& v_{1}=N /\|N\|_{2} \\
& \text { for } j=1,2, \ldots, n \text { do } \\
& \text { for } i=1,2, \ldots, j \text { do } \\
& h_{i j}=\left(M v_{j}, v_{i}\right) \\
& \text { end for } \\
& w_{j}=M v_{j}-\sum_{i=1}^{j} h_{i j} v_{i} \\
& h_{j+1, j}=\left\|w_{j}\right\|_{2} \\
& \text { if } h_{j+1, j}=0, \text { then Stop } \\
& v_{j+1}=w_{j} / h_{j+1, j} \\
& \text { end for }
\end{aligned}
$$

A second product of the Arnoldi iteration is the rectangular Hessenberg matrix $\boldsymbol{H}_{n} \in \mathbb{R}^{(\mathrm{n}+1) \times \mathrm{n}}$. If breakdown occurs in the $n$-th step, $\mathrm{w}_{\mathrm{n}}=0$ is still well-defined but not $\mathbf{v}_{\mathbf{n}+\mathbf{1}}$, and the algorithm stops. In this case the last row of $\boldsymbol{H}_{\boldsymbol{n}}$ is set to zero, $h_{n+1}=0$.

\subsection{Coupling of reduced components}

The assembly of reduced substructures, typically a set of valves, dead legs, and header, is outlined in this section. In the following, $r$ denotes the order of the reduced order models and $n$ denotes the number of reduced substructures being coupled together.

Any reduced order model of a substructure may be written as

$$
\boldsymbol{C}^{(s)} \boldsymbol{V} \dot{\boldsymbol{\tau}}^{(s)}(t)+\boldsymbol{K}^{(s)} \boldsymbol{V} \boldsymbol{\tau}^{(s)}(t)=\boldsymbol{q}^{(s)} u(t)+\boldsymbol{g}^{(s)}+\boldsymbol{R}^{(s)} .
$$

$\mathrm{g}^{(\mathrm{s})} \in \mathbb{R}^{\mathrm{N}}$ is the vector of connection fluxes between substructure $\mathrm{s}$ and its direct neighbours, and $\mathbf{R}^{(s)}$ is the vector of reaction forces between substructures and its neighbours. Multiplying on the left by $\boldsymbol{V}^{T}$,

$$
\boldsymbol{V}^{T} \boldsymbol{C}^{(s)} \boldsymbol{V} \dot{\tau}^{(s)}(t)+\boldsymbol{V}^{T} \boldsymbol{K}^{(s)} \boldsymbol{V} \boldsymbol{\tau}^{(s)}(t)=\boldsymbol{V}^{T} \boldsymbol{q}^{(s)} u(t)+\boldsymbol{V}^{T} \boldsymbol{g}^{(s)}+\boldsymbol{V}^{T} \boldsymbol{R}^{(s)} .
$$

the term $\boldsymbol{V}^{T} \boldsymbol{R}^{(s)}$ is enforced to be equal to 0 , following from the nature of the projection matrix $\boldsymbol{V}^{T}$ with is a Petrov-Galerkin projector. It follows that the coupled model of $n$ substructures is expressed as

$$
\begin{array}{clc}
\boldsymbol{C}_{r} \dot{\boldsymbol{\tau}}+\boldsymbol{K}_{r} \boldsymbol{\tau} & =\boldsymbol{q}_{r}+\boldsymbol{g}_{r} \\
\boldsymbol{C}_{\boldsymbol{r}} & =\boldsymbol{V}^{T} \boldsymbol{C} \boldsymbol{V} \\
\boldsymbol{K}_{\boldsymbol{r}} & =\boldsymbol{V}^{T} \boldsymbol{K} \boldsymbol{V} \\
\boldsymbol{q}_{\boldsymbol{r}} & =\boldsymbol{V}^{T} \boldsymbol{q} \\
\boldsymbol{g}_{r} & =\boldsymbol{V}^{T} \boldsymbol{g}
\end{array}
$$

At the interface between neighboring coupled substructures, compatibility of nodal temperatures and conservation of heat leads to two constraints on the nodal temperatures, (12), and heat fluxes at the substructure boundary (13) 


$$
\begin{gathered}
\boldsymbol{B}_{\boldsymbol{r}} \boldsymbol{\tau}=0, \\
\boldsymbol{L}_{\boldsymbol{r}}^{\boldsymbol{T}} \boldsymbol{g}_{\boldsymbol{r}}=0
\end{gathered}
$$

In (12) matrix $\boldsymbol{B}_{\boldsymbol{r}} \in \mathbb{R}^{\mathrm{b}}$ is a signed Boolean matrix in the case of conformal boundary between substructure $S$ and its neighbors. If the interface is not conforming, $\boldsymbol{B}_{\boldsymbol{r}}$ is a general matrix. Similarly, in (13) matrix $\boldsymbol{L}_{\boldsymbol{r}}$ imposes that the sum of nodal fluxes at the interface is zero, be that in the case of a conformal or general interface. A discussion and detailed explanation of the formation of these coupling matrices is found in the appendix of reference [2]. Only a general overview is given in the following.

The unique generalized coordinates $\varphi$ satisfying the compatibility condition are found as

which leads to

$$
L_{r} \varphi=\tau,
$$

$$
B_{r} \tau=B_{r} L_{r} \varphi=0 \quad \forall \varphi .
$$

It follows that $\mathrm{L}_{\mathrm{r}}$ is the kernel of $\mathrm{B}_{\mathrm{r}}$.

The global coupled model of $\mathrm{n}$ substructures has therefore the following form

$$
\begin{array}{ccc}
\boldsymbol{C}_{\boldsymbol{r}} \dot{\boldsymbol{\tau}}+\boldsymbol{K}_{r} \boldsymbol{\tau} & = & \boldsymbol{q}_{r}+\boldsymbol{g}_{\boldsymbol{r}} \\
\boldsymbol{B}_{\boldsymbol{r}} \boldsymbol{\tau} & = & \mathbf{0} \\
\boldsymbol{L}_{\boldsymbol{r}}^{\boldsymbol{T}} \boldsymbol{g}_{r} & = & \mathbf{0}
\end{array}
$$

The equilibrium condition is imposed only weakly, in the sense that

$$
L_{r}^{T} g_{r}=L_{r}^{T} V^{T} g=0 .
$$

The global assembled reduced system using unique generalized coordinates have then the form of

$$
\begin{array}{ccc}
\widetilde{\boldsymbol{C}_{r}} \dot{\boldsymbol{\varphi}}+\widetilde{\boldsymbol{K}_{r}} \boldsymbol{\varphi} & =\widetilde{\boldsymbol{q}_{r}} \\
\widetilde{\boldsymbol{C}_{r}} & = & L_{r}^{T} C_{r} L_{r} \\
\widetilde{\boldsymbol{K}_{r}} & = & L_{r}^{T} K_{r} L_{r} \\
\widetilde{\boldsymbol{q}_{r}} & = & L_{r}^{T} q_{r}
\end{array}
$$

The global assembled reduced system of equations describes a reduced order approximation of the global assembled system of equations. The quality of the approximation is given by the model order reduction method put to use. The selection of suitable model order reduction method is crucial in making the approximation reasonably small.

Substructuring allows division of the computation domain into smaller parts in a framework allowing coupling at a later stage. It also enables coupling between substructures belonging to different assemblies, provided that the interface is conforming. However, the main contribution of substructuring lies in the possibility of coupling reduced structures. It is much more computationally effective to reduce a set of small substructures than to reduce the whole structure. It is also straightforward to parallelize the computation of a large number of substructures making it again much more efficient. Another advantage is the possibility of coupling structures made at different stages of the project work, possibly by different persons. 


\subsection{Solution procedure}

The solution procedure consists of the following steps:

1. Model order reduction of substructures;

2. Coupling of reduced order models of each substructure with each other into a complete reduced order model;

3. Steady-state analysis;

4. Transfer of the obtained temperature distribution to the model used for cool down analysis;

5. Transient analysis of the cool down;

6. Transformation to physical coordinates;

7. Postprocessing.

A reduced-order model of each substructure, following the procedure outlined in section 3.1, is generated. The substructures need not be in the same coordinate system. The Arnoldi algorithm used to generate the projection matrix on a Krylov subspace characterizing the original LTI system is implemented in ANSYS APDL. Reordering of matrices and other necessary processing was implemented in MATLAB 12a. The output of the Arnoldi algorithm is the projector matrix $\boldsymbol{V}$ for each substructure. This projection matrix is then used to reduce the system matrices to arrive at (11). All reduced models need then to be coupled and assembled into a single reduced order model of the discretized heat transfer equations in generalized coordinates. The model can then be solved to a steadystate solution, which is then used as initial condition to a transient cool-down analysis performed with help of a four step Runge-Kutta algorithm. In (2) we have considered only zero initial conditions. To zero out the initial conditions this transformation is used

$$
\begin{gathered}
T(t)=\tilde{T}(t)+T_{0}, \\
\dot{T}(t)=\tilde{\tilde{T}}(t) .
\end{gathered}
$$

Then in new coordinates $\tilde{T}(t)$ the initial conditions are $\tilde{T}(0)=0$, and system $(2)$ reads

$$
\begin{gathered}
C \dot{\tilde{T}}+K \tilde{T}=Q-K T_{0} \\
\tilde{T}(0)=0 .
\end{gathered}
$$

The transformation to physical coordinates with help of (4) can then be performed, followed by postprocessing of the obtained solution.

\section{Results}

In this section two sets of results are presented. The first set consists of computational times and cool down curves obtained by a conventional finite element method, where the discretization in time is performed on a full model. The second set consists of the computational times and cool down curves obtained with a model consisting of coupled reduced substructures. 


\subsection{Steady state solution}

The steady state solution of the full model was conducted in ANSYS APDL, on a model with $32.6 \times 10^{6}$ nodes. The solution took about 2.5 hours. The resulting temperature field was then transferred to the cool down simulation, section 4.2 , as initial condition.

Steady state solution of the reduced order model was conducted in MATLAB, within $0.2 \mathrm{~s}$. The resulting temperature field was then transferred to the reduced order model obtained for the steady state boundary conditions, see section 2 . Generation of the reduced order models necessitated 0.8 hours.

\subsection{Transient solution}

The transient solution of the full model with production shutdown and with initial condition from the steady state solution was run for a total duration of 13 hours, with two time stepping strategies:

1. Initial time step of $1 \mathrm{~s}$, with a minimum time step of $1 \mathrm{~s}$ and a maximum time step of 3600 s;

2. Initial time step of $1 \mathrm{~s}$, with a minimum time step of $1 \mathrm{~s}$ and a maximum time step of $900 \mathrm{~s}$.

The machine run times for time stepping strategies 1 and 2 were 72 hours and 121 hours, respectively, with time integration performed by generalized trapezoidal rule.

On the reduced order model, the cool down simulation necessitated $10 \mathrm{~s}$. The time step used in the transient solution of the reduced order model was $1 \mathrm{~s}$, for a total simulated duration of 13 hours.

\section{Discussion}

The convergence of model order reduction is captured in Figure 3. The graph compares the cool down of the manifold simulated using the full model in ANSYS for the second time stepping strategy presented in section 4.2 against the simulations using Krylov model order reduction. The transient solution is shown for one nodal temperature in the model. Krylov model order reduction is presented for orders of reduction $n=4,10,20$. The transient simulation was run in MATLAB using the ode15s solver.

The graph shows very good agreement between reduced model simulations and the full model simulation. Difference between reduced models and full model at the end of the simulation is $0.08^{\circ} \mathrm{C}, 0.07^{\circ} \mathrm{C}, 0.07^{\circ} \mathrm{C}$ for reduction orders $n=4,10$ and 20 respectively. At the end of the simulations the reduced order models have lower temperature, which in the context of flow assurance calculations is acceptable.

The solution obtained with a reduction order of 4 exhibits slight deviation at the beginning of the simulation, the error being less than $0.2^{\circ} \mathrm{C}$. This error vanishes 
with time so even reduced models of order 4 may serve as good approximation of the full model. In order to assess the reduction model thoroughly following error metric $\varepsilon$ is suggested

$$
\varepsilon=\max _{i}\left(\max \left|\boldsymbol{T}_{i}-\boldsymbol{V} \boldsymbol{\tau}_{\boldsymbol{i}}\right|\right)
$$

where $\boldsymbol{T}_{i}$ is the vector of temperatures from the full model simulation at time step $i$ and $\tau_{i}$ is vector of generalized temperature from the reduced order model simulation at time step $i . \boldsymbol{V}$ is the projection matrix. The error metric is the maximum of the maximum value of the absolute difference between temperatures of the full model and the expanded reduced order model. For the manifold the value of the error metric is $\varepsilon=0.16^{\circ} \mathrm{C}$ for a reduced model of order 20 . The low value of error metric implies very good overall approximation of the full model by a reduced order model. Simulation of manifold cool down with model order reduction is faster by more than 10 times compared to the cool down simulation of the full model in ANSYS.

There are two simulations - steady state and transient. Hence, it is necessary to reduce the substructure for each simulation. Any change in the boundary condition leads to the need of a new reducing step for each substructure.

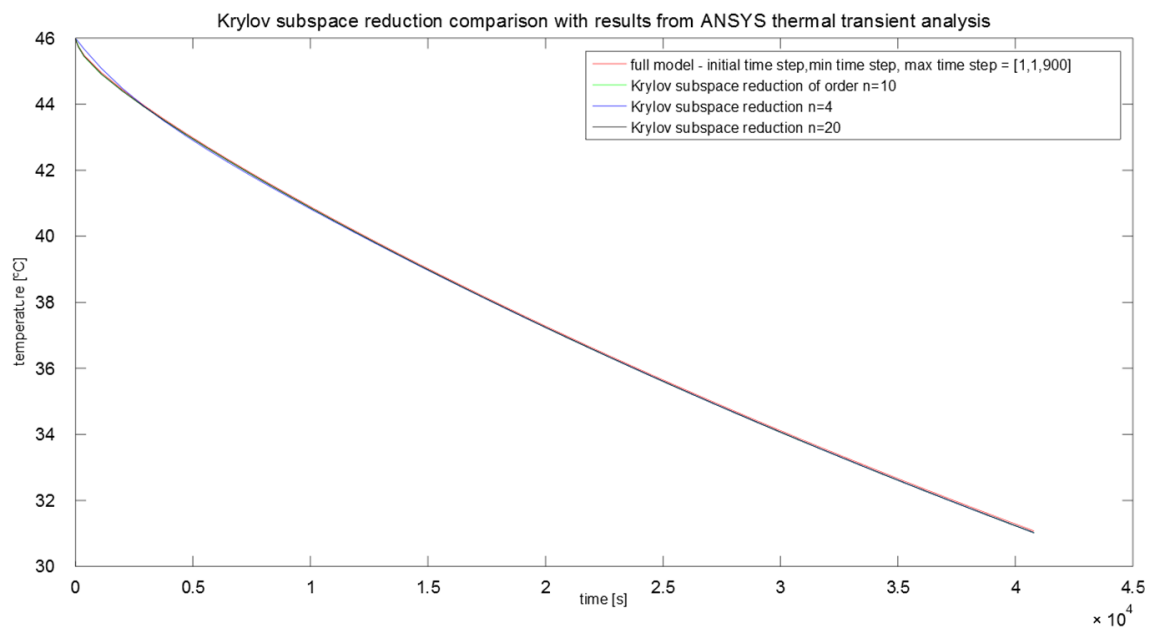

Figure 3: Transient simulation times and profile for the full model solved using implicit time stepping and reduced model with reduction order 4, 10, 20 advanced in time by a four-step Runge Kutta algorithm.

\section{Conclusions}

A methodology integrating the use of substructuring and model order reduction for the fast simulation of the thermal steady state and transient solutions of subsea equipment has been established. 
The heat transfer by convection in the production fluid is mimicked in this work by the use of a high thermal conductivity. This does not detract from the method itself, but ongoing work aims at removing this limitation by extension of model order reduction by projection on a Krylov subspace to nonlinear convective transport.

Further work involves the development of methods allowing the reduction of models independently of the initial conditions, and valid for a wide range of boundary conditions. This type of development is called PMOR, as named in the introduction to this paper.

\section{References}

[1] D. de Klerk, D. J. Rixen, and S. N. Voormeren, General framework for dynamic substructuring: History, review, and classification of techniques, AIAA Journal, vol. 46, no. 5, May 2008.

[2] A. C. Antoulas. Approximation of large-scale dynamical systems. Advances in Design and Control, SIAM, Philadelphia, 2005.

[3] Roland W. Freund. Krylov-subspace methods for reduced-order modeling in circuit simulation. Journal of Computational and Applied Mathematics, 123:395-421, 2000.

[4] E. J. Grimme. Krylov projection methods for model reduction. PhD thesis, Uni. Illinois at Urbana Champaign, 1997.

[5] P. Koutsovasilis and M. Beitelschmidt, Comparison of model reduction techniques for large mechanical systems, Multibody System Dynamics, vol. 20, pp. 111-128, 2008.

[6] W. Witteveen, Comparison of CMS, Krylov and Balanced Truncation Based Model Reduction from a Mechanical Application Engineer's Perspective, in Topics in Experimental Dynamics Substructuring and Wind Turbine Dynamics, Volume 2, vol. 27, R. Mayes, D. Rixen, D. Griffith, D. De Klerk, S. Chauhan, S. Voormeeren and M. Allen, Eds., Springer New York, pp. 319-331, 2012.

[7] U. Baur, C. Beattie, P. Benner and S. Gugercin, Interpolatory Projection Methods for Parameterized Model Reduction, SIAM J. Sci. Comput., vol. 33, no. 5, pp. 2489-2518, Oct 2011.

[8] J. Šindler, A. Suleng, T. Jelstad Olsen and P. Bárta, Krylov Model Order Reduction of a Thermal Subsea Model, World Academy of Science, Engineering and Technology, vol. 077, pp. 217-224, May 2013.

[9] L. H. Feng, E. B. Rudnyi, J. G. Korvink, C. Bohm and T. Hauck, Compact Electro-thermal Model of Semiconductor Device with Nonlinear Convection Coefficient, in In: Thermal, Mechanical and Multi-Physics Simulation and Experiments in Micro-Electronics and Micro-Systems. Proceedings of EuroSimE 2005, 2005.

[10] Peter Benner, Tobias Breiten, Krylov-Subspace Based Model Reduction of Nonlinear Circuit Models Using Bilinear and Quadratic-Linear Approximations, Progress in Industrial Mathematics at ECMI 2010 Mathematics in Industry, pp 153-159, 2012. 
188 Boundary Elements and Other Mesh Reduction Methods XXXVII

[11] A. Suleng, T. Jelstad Olsen, J. Šindler, P. Bárta, Tuning of Thermal FEA Using Krylov Parametric MOR for Subsea Application, World Academy of Science, Engineering and Technology, 2012. 